\title{
Reduced expression of mir15a in the blood of patients with oral squamous cell carcinoma is associated with tumor staging
}

\author{
JOÃO ARTUR RICIERI BRITO ${ }^{1}$, CAROLINA CAVALIÉRI GOMES ${ }^{1}$, \\ FLÁVIO JULIANO GARCIA SANTOS PIMENTA ${ }^{1}$, ALVIMAR AFONSO BARBOSA ${ }^{2}$, \\ MARCO ANTÔNIO MÁXIMO PRADO ${ }^{4}$, VÂNIA FERREIRA PRADO ${ }^{4}$, \\ MARCUS VINÍCIUS GOMEZ ${ }^{3}$ and RICARDO SANTIAGO GOMEZ ${ }^{1}$

\begin{abstract}
${ }^{1}$ Department of Oral Surgery and Pathology, School of Dentistry, ${ }^{2}$ Department of Surgery, School of Medicine, and ${ }^{3}$ School of Medicine, Universidade Federal de Minas Gerais, Belo Horizonte, Brazil; ${ }^{4}$ Robarts Research Institute and Departments of Physiology and
\end{abstract} \\ Pharmacology/Anatomy and Cell Biology, University of Western Ontario, Ontario, Canada
}

Received September 2, 2009; Accepted October 19, 2009

DOI: 10.3892/etm_00000035

\begin{abstract}
MicroRNAs (miRNAs) mir15a and let7a are important regulators of bcl-2, ras and c-myc proteins. Considering that these miRNAs are commonly altered in many human cancers and that these proteins are reported to be altered in oral squamous cell carcinoma (OSCC), we investigated them in a set of OSCC cases. The miRNAs as well as the proteins were evaluated in the tumor and blood of 20 patients by real-time quantitative PCR and immunohistochemistry, respectively. The expression of mir15a and bcl-2 proteins in the tumors was not associated with each other or with tumor staging. On the other hand, we found reduced expression of this miRNA in the blood of patients with an advanced stage of OSCC and with lymph node metastasis. The expression of let7a in the tumor and blood was not associated with tumor size, lymph node metastasis, tumor staging and immunoexpression of ras and c-myc proteins. In conclusion, the present study shows that reduced expression of mir15a is associated with OSCC staging.
\end{abstract}

\section{Introduction}

MicroRNAs (miRNAs) are small non-coding evolutionarily conserved RNAs which mediate gene expression at the post-transcriptional level by degrading or repressing target messenger RNA (mRNA) $(1,2)$. miRNAs are products

Correspondence to: Dr Ricardo Santiago Gomez, Departmento de Clínica, Patologia e Cirurgia, Faculdade de Odontologia, Universidade Federal de Minas Gerais, Av. Antonio Carlos 6627, Belo Horizonte CEP 31270 901, Brazil

E-mail: rsgomez@ufmg.br

Key words: oral cancer, microRNA, mir15a, let7a approximately 22 nucleotides long which regulate mRNA translational by base pairing to partially complementary sites, predominantly in the $3^{\prime}$ untranslated region (3'UTR) (2). Each miRNA has the potential to regulate many target genes in humans, modulating the levels of thousands of mRNAs, which implies that over one third to one half of all protein-encoding genes in humans are regulated by miRNAs (3). Considering the important effect of miRNAs on gene expression, it is not surprising that these small RNAs have been implicated in the pathogenesis of cancer (4). The expression profiles of miRNAs are usually altered in many cancers. A reduction in miRNAs accelerates oncogenic transformation through the deregulation of target oncogenes (5). Oncogenic miRNAs have also been described and have been shown to be involved in the pathogenesis of some tumors. In this case, the increased transcription of miRNA inactivates a tumor-suppressor gene.

Oral squamous cell carcinoma (OSCC) is one of the most frequent cancers in the world, and its main risk factors include smoking and alcohol consumption. This neoplasia results from multiple genetic events, resulting in damage to signalling pathways and the regulation of the cell cycle. The 5-year survival rate for cancers of the tongue, oral cavity and oropharynx is approximately $50 \%$ (6). Despite the importance of miRNAs in human cancer, few studies have attempted to evaluate their expression in oral cancer (7). In the present study, we selected two miRNAs (mir15a and let7a) that are commonly altered in different types of cancer. We also chose these miRNAs as they regulate the expression of proteins reported to be altered in OSCC (bcl-2, ras and c-myc). The results showed decreased expression of mir15a in the blood of patients with advanced stage OSCC and lymph node metastasis.

\section{Materials and methods}

A total of 20 primary tumors and peripheral blood were obtained from 20 patients with OSCC immediately after 
surgical resection. The average age of the patients was 57 years (range, 37-90 years), and they were predominantly male. Histological classification was performed according to the International Histological Classification of Tumors (8) and the TNM staging system according to the International Union Against Cancer (UICC).

The tissue samples were collected in RNAholder (BioAgency, Sao Paulo, Brazil). All samples were collected at the time of surgery. A section of the lesion was immediately snap frozen and stored at $-80^{\circ} \mathrm{C}$. The other part was fixed in buffered formalin for histopathological diagnosis. Histopathological analysis confirmed that each tissue sample had a $>60 \%$ tumor cell content. The peripheral blood $(4 \mathrm{ml})$ was collected immediately after surgical resection of the primary tumor. The blood was collected in a tube with anticoagulant (EDTA) and stored at $-80^{\circ} \mathrm{C}$ until processing. Fragments of normal oral mucosa and the peripheral blood of healthy volunteers were collected during non-neoplastic or preprosthetic surgical procedures and were used as control. The present study was approved by the local ethics committee, and a signed informed consent was obtained from all of the patients as well as the healthy volunteers.

Total RNA was isolated from $25 \mathrm{mg}$ of human frozen samples and $250 \mu \mathrm{l}$ of peripheral blood sample in $1 \mathrm{ml}$ of TRIzol Reagent (Invitrogen Life Technologies, Inc., Carlsbad, CA, USA), according to the manufacturer's instructions. The RNA was briefly treated with RNAse-free DNAase I (Invitrogen Life Technologies), and the cDNA was synthetized from $1 \mu \mathrm{g}$ of total RNA using gene-specific stem-loop primers to miRNA (9) (TaqMan MicroRNA RT; Applied Biosystems, Foster City, CA, USA). The expression of miRNA was profiled using a real-time quantitative TaqMan assay (TaqMan ${ }^{\circledR}$ MicroRNA Assays; Applied Biosystems) in a Step-One real-time PCR 48-well plate (Applied Biosystems). The $20 \mu \mathrm{l}$ total reaction included $2 \mu \mathrm{l}$ of cDNA, $2 \mathrm{X}$ TaqMan Universal PCR master mix (no EmpErase UNG) and probe mix of the TaqMan MicroRNA Assay protocol (Applied Biosystems). The PCR triplicate reaction was performed in a 48 -well optical plate at $95^{\circ} \mathrm{C}$ for $10 \mathrm{~min}$, followed by $15 \mathrm{sec}$ at $95^{\circ} \mathrm{C}$ and $1 \mathrm{~min}$ at $60^{\circ} \mathrm{C}$ for 40 cycles. The mean of the threshold cycle $(\mathrm{Ct})$, defined as the fractional cycle number at which the fluorescence passes the fixed threshold, was determined using default threshold settings. Relative gene expression was calculated using the $2^{-\Delta \Delta \mathrm{Ct}}$ method (Applied Biosystems User Bulletin No. 2) as previously described (10), and the expression data were normalized with endogenous miRNA RNU48, U47 and RNU44. The data were presented as $\log 10$ of the relative quantity of target miRNA normalized to endogenous miRNA and relative to a calibrator sample. As calibrator we used a pool of normal oral tissues for the tumoral tissues, and blood samples of healthy individuals for the blood of patients with OSCC.

The total RNA input ranged from 6.25 to $100 \mathrm{ng}$. The results showed that the method was able to detect miRNA in as little as 10 or $100 \mathrm{ng}$ of total RNA. As the amount of cDNA was decreased, the amplification started later, giving a higher $\mathrm{Ct}$. We used $10 \mathrm{ng}$ of RNA for all experiment to obtain a good balance between cDNA economy and an assay of good quality. For the let7a, the slope value was -3.329 and $\mathrm{R}^{2}$ was 0.979 . The mir15a experiments showed a slope value of -3.28 and $\mathrm{R}^{2}$ of 0.987 . The endogenous control RNU44, U47 and RNU48 showed similar values (slope $=-3.33$ and $R^{2}=0.981$ ).

The following antibodies were used for immunohistochemical analysis: bcl-2 (diluted 1:20, Clone 124; Dako ${ }^{\circledR}$ Corp., Carpinteria, CA, USA), c-myc (diluted 1:75, Clone 3C117; Santa Cruz ${ }^{\circledR}$ Biotechnology Inc., Santa Cruz, CA, USA) and h-ras (diluted 1:20, Clone F235; Santa Cruz Biotechnology). Immunohistochemical stains were performed using the high sensitive polymer-based system (EnVision; Dako Corp.) with diaminobenzidine substrate solution as chromogen (Sigma, St Louis, MO, USA). In the immunohistological analysis, only sections containing sufficient neoplasic epithelium were used to assess the antibody reactivity. Two experienced independent pathologists examined multiple fields and scored the tissue sections for the extent of staining, regardless of staining intensity (score 0 , negative staining; score 1, 0-25\% of positive cells; score $2,25-50 \%$ of positive cells; score $3,>50 \%$ of positive cells).

Statistical methods. Non-parametric Spearman correlation coefficients were used to assess the association between continuous variables. The non-parametric Mann-Whitney test was used to compare two groups of cases on one variable. Statistical analyses were performed using BioStat 4.0 software (Optical Digital Optical Technology, Belém, Brazil), and a p-value $\leq 0.05$ was considered statistically significant.

\section{Results}

The gender, age, location, TNM, tumor staging and miRNA expression in the tumor and blood of patients with OSCC are presented in Table I.

Expression of mirl5a in OSCC. Compared to normal oral tissues, increased expression of mir15a was found in 14 cases of OSCC (87.5\%). The median level of mir15 expression in the tumors of early stages (I, II and III) was not different when compared with that of tumors in an advanced stage (Table I). No statistical difference was found according to tumor size and lymph node metastasis.

Expression of mirl5a in the blood samples. While decreased expression of mir15a was found in the blood samples of 6 patients with OSCC (35.2\%), increased expression was observed in 11 cases $(64.8 \%)$ (Table I). All of the cases with decreased expression of mir15a in the blood were OSCC cases in an advanced stage (stage IV) with lymph node metastasis. Therefore, reduced expression of this miRNA in the blood was associated with an advanced stage of the tumor $(\mathrm{p}=0.006)$ (Fig. 1) and with lymph node metastasis $(\mathrm{p}=0.010)$ (Fig. 2). The levels of mir15a expression in the blood samples of patients with OSCC were clearly lower than the levels in the matched tumor samples $(\mathrm{p}=0.034)$.

mirl5a and bcl-2 protein expression. Only sections containing sufficient neoplasic epithelium were examined. Positive labeling for bcl-2 protein was observed in 4 samples of OSCC (score 1). The expression of mir15a in the cases with negative immunostaining for bcl-2 was not statistically different compared to the lesions with positive labeling. 
Table I. Gender, age, location, TNM, tumor staging, let7a and mir15a miRNA expression in the blood and tumors of oral squamous cell carcinoma patients included in the study.

\begin{tabular}{|c|c|c|c|c|c|c|c|c|c|}
\hline \multirow[t]{2}{*}{ Case } & \multirow[t]{2}{*}{ Gender } & \multirow[t]{2}{*}{ Age } & \multirow[t]{2}{*}{ Location } & \multirow[t]{2}{*}{ TNM } & \multirow[t]{2}{*}{ Stage } & \multicolumn{2}{|c|}{ Let7a expression ${ }^{a}$} & \multicolumn{2}{|c|}{ mir15a expression $^{a}$} \\
\hline & & & & & & Blood & Tumor & Blood & Tumor \\
\hline 1 & M & 56 & Retromolar trigone & $\mathrm{T} 2 \mathrm{~N} 2 \mathrm{M} 0$ & IV & 1.119 & 0.512 & 0.490 & 3.944 \\
\hline 2 & M & 55 & Floor of the mouth & T3N0M0 & III & 0.864 & 2.506 & 10.446 & - \\
\hline 3 & $\mathrm{~F}$ & 61 & Tongue & T4N2M0 & IV & 0.784 & 4.075 & 0.129 & 12.554 \\
\hline 4 & M & 49 & Floor of the mouth & T3N0M0 & III & 4.662 & 0.713 & 0.462 & - \\
\hline 5 & M & 51 & Tongue & T2N0M0 & II & 3.969 & 1.289 & 1.543 & 3.144 \\
\hline 6 & $\mathrm{~F}$ & 68 & Tongue & T4N1M0 & IV & - & 0.004 & - & 3.683 \\
\hline 7 & M & 56 & Retromolar trigone & T4N0M0 & IV & - & 0.782 & - & - \\
\hline 8 & $\mathrm{~F}$ & 41 & Tongue & T3N0M0 & III & 0.020 & 1.221 & 0.546 & 5.579 \\
\hline 9 & M & 59 & Gingiva + floor of the mouth & T4N0M0 & IV & 0.595 & 7.705 & 0.249 & 1.965 \\
\hline 10 & M & 55 & Retromolar trigone & T4N1M0 & IV & 0.003 & 41.144 & 0.005 & 0.032 \\
\hline 11 & M & 44 & Floor of the mouth & T4N0M0 & IV & 0.557 & 2.370 & 2.084 & 18.466 \\
\hline 12 & M & 37 & Floor of the mouth & T4N0M0 & IV & 0.001 & 1.448 & 0.599 & 1265.276 \\
\hline 13 & M & 59 & Tongue & T2N0M0 & II & 0.079 & 0.634 & 3.693 & 14.739 \\
\hline 14 & M & 74 & Gingiva & T4M0M0 & IV & 6.545 & 1.265 & 2.319 & 1.568 \\
\hline 15 & $\mathrm{~F}$ & 50 & Floor of the mouth & T2N0M0 & II & 4.509 & 0.403 & 4.084 & 3.498 \\
\hline 16 & M & 73 & Retromolar trigone & T2N0M0 & II & 0.934 & 0.388 & 4.029 & 0.220 \\
\hline 17 & M & 72 & Floor of the mouth & T4N3M0 & IV & 0.488 & 8.066 & 0.429 & 162.979 \\
\hline 18 & M & 61 & Floor of the mouth & T2N0M0 & II & 4.415 & 41.087 & 2.134 & 226.736 \\
\hline 19 & M & 68 & Tongue + retromolar trigone & T3N0M0 & III & 3.236 & 1.495 & 121.275 & 5.499 \\
\hline 20 & M & 73 & Gingiva & T4N0M0 & IV & 8.196 & 34.967 & 3.517 & 325.293 \\
\hline
\end{tabular}

a $2-\Delta \Delta \mathrm{Ct}$

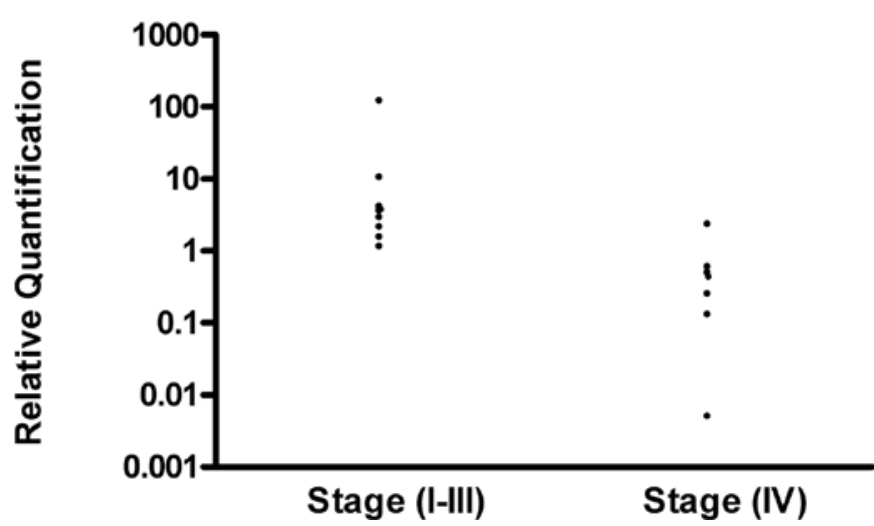

Figure 1. Relative quantification $\left(2^{-\Delta \Delta C t}\right)$ of mir15a in oral squamous cell carcinoma stage I-III compared to stage IV. Decreased mir15a expression was noted in the tumors with stage IV $(\mathrm{p}=0.006)$.

Expression of let7a in OSCC. While higher levels of let7a expression in OSCC compared to normal oral mucosa were found in 13 cases $(61.9 \%)$, decreased expression was found in 18 cases $(38.1 \%$ ) (Table I). The expression of let7a was not associated with tumor size, lymph node metastasis and tumor stage.

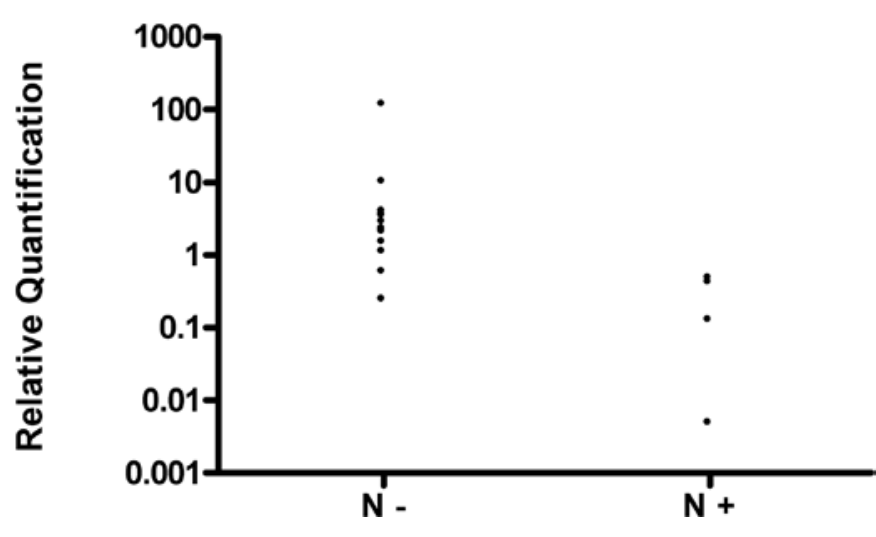

Figure 2. Relative quantification $\left(2^{-\Delta \Delta \mathrm{Ct}}\right)$ of mir15a in oral squamous cell carcinoma without lymph node metastasis $(\mathrm{N}-)$ compared with positive lymph node metastasis $(\mathrm{N}+)$. Decreased mir15a expression was noted in the $\mathrm{N}+$ cases $(\mathrm{p}=0.010)$.

Expression of let7a in blood samples. Decreased expression of let7a was found in the majority (18 out of 20) of blood samples of patients with OSCC (Table I). The expression of let7a in the blood was significantly lower than that in the matched tumors. No statistical association was found regarding lymph node metastasis and tumor size. No statistical correlation was found between the expression of let7a and mir15a. 
Let7a and c-myc or ras protein expression in OSCC. Most of the samples (14 out of 20) presented moderate (score 2) or strong (score 3) c-myc expression. The expression of let7a in the cases with c-myc score 2 was not statistically different when compared to the expression of let7a in the cases with c-myc score 3. All cases with decreased expression of let7a showed strong positive staining for c-myc, but this association was not statistically significant.

Most of the samples were positive for the ras protein. The expression of let7a in the cases with ras staining score 1 was not statistically different when compared to the expression of let7a in the cases with ras staining score 3.

Expression of bcl-2, c-myc and ras regarding tumor staging. No relationship was observed between expression of bcl-2, c-myc and ras with tumor staging.

\section{Discussion}

Tran et al (11) investigated 261 mature miRNA genes in nine head and neck cancer cell lines using an oligonucleotide array platform. The cell lines were from carcinomas of the hypopharynge, tongue, larynx and tonsil. The authors found overexpression of 33 miRNAs, and 22 exhibited low expression. Another study showed that cancer cell lines from head and neck overexpress miR-205r (12). One important limitation of these studies was the use of cancer cell lines for investigation. miRNA from cancer cell lines may not show the same profile as the original tumor. According to some researchers, in vitro growth conditions including the inclusion of synthetic medium and fetal bovine serum may have an effect on miRNA expression patterns in human tumor cell lines (13).

Evidence suggests that miRNAs may control cancer development, or at least play a critical role in the pathogenesis through regulation of cell growth and apoptosis. The association of miRNAs with the expression of important oncogenes such as bcl-2, c-myc and ras supports a key role for these small molecules in human cancer.

Diminished or loss of expression of mir15a has been demonstrated in chronic lymphocytic leukemia and in prostate cancer (14). The 3'UTR of the bcl-2 transcript has potential binding sites for mir15a and mirl6-1, and these miRNAs have the ability to downregulate the anti-apoptotic protein bcl-2 (15). Furthermore, the expression of this miRNA induces apoptosis in leukemia cell lines. Considering that OSCC shows altered expression of bcl-2 (16) we attempted to investigate the association between this miRNA and its target protein, together with its impact on clinical staging. We observed increased expression of mir15a in most of the OSCC cases, which may be associated with the negative staining found for bcl-2 in many of the samples studied. However, we did not observe any statistical significant association between this miRNA and this anti-apoptotic protein. The lack of association between both may be explained by other proteins or regulators of bcl-2 apart from mir15a which are also able to influence bcl-2 expression. For example, bcl-2 expression may be regulated by tyrosine kinase inhibitors that can regulate apoptosis and inhibit bcl-2 expression. bcl-2 phosporylation may be another regulatory mechanism of bcl-2 protein expression (17).
We did not find an association between mir15a expression in OSCC and tumor staging, tumor size and lymph node metastasis. Notably, reduced expression of mir15a was found in the blood of patients with lymph node metastasis or in advanced clinical staging (stage IV) compared to stages I, II and III. Loss of heterozygosity of 13q.14.3, the same locus of mir15a, was previously reported in OSCC and was correlated with lymph node metastasis (18). These data further support the importance of chromosome $13 q$ in the pathogenesis of OSCC. These findings also suggest that the expression of this tumor-supressor miRNA in the blood of patients with OSCC may also be useful in tumor staging.

Considering the methodology used in the present study, it is not possible to know the origin of mir15a found in the blood. The possible sources of this miRNA include circulating leukocytes, cancer cells or their products that have reached the circulation. Although the decreased expression of this miRNA may have an impact in the expression of bcl-2 in potentially metastatic cancer cells in the blood, this hypothesis needs to be tested.

We did not find an association between let7a expression and OSCC staging. Reduced expression of let7a was significantly associated with shortened postoperative survival in patients with lung cancer. Furthermore, let7a overexpression results in the inhibition of lung cancer cell growth (19), as well as the growth of human colon cancer cells (20). Recent studies have shown an association between miRNA expression, such as let7 and mir-205, and the severity and poor survival in patients with head and neck cancer $(21,22)$. Our data did not confirm a tumor-suppressor role for let7a in the pathogenesis of OSCC.

Let7 miRNA negatively regulates the expression of ras and c-myc by targeting their mRNAs for translation repression (23). In lung cancer, reduced levels of let7 were observed together with increased expression of the ras protein (19). The presence of any ras variant was significantly associated with poor prognosis in squamous cell carcinoma of the head and neck, and prognosis was worse among cases of oral cancer (24). Studies of ras expression in squamous cell carcinoma of the head and neck have indicated that amplified ras promotes growth, and the presence of the ras protein has been associated with late stage tumors and increased tumor size (25). Most of the OSCC cases in the present study were positive for the ras protein, but the expression of this protein was not associated with let7 expression or with tumor size or lymph node metastasis.

Overexpression of c-myc has been observed in 20-40\% of OSCC cases. Overexpression of c-myc provides changes in the regulation of many genes and contributes to malignant transformation (26). In our study, we observed moderate or strong c-myc expression in most of the samples studied. The amplification status of the myc gene has often been studied in parallel with ras. H-ras mutations have been described in OSCC, but the exact mechanism accounting for ras overexpression is unknown, and its association with existing prognostic factors is still unclear. A high level of c-myc expression is associated with shortened overall survival, tumor size and clinical stage $(26,27)$. In our study no relationship was found between c-myc protein and tumor staging. As we did not find an association between let7a expression with ras or 
c-myc protein immunolocalization, other genetic or epigenetic alterations may be more relevant to the expression of these oncogenes in OSCC.

Although the findings here reported are informative, the phenomenon of genomic convergence (clonal adaptation) should be considered in the critical analysis of the data. According to this theory, early stage tumors are karyotypically heterogeneous, while late stage tumors are often found to be karyotypically homogeneous due to selection pressures (28). Therefore, it is not possible to know which of the lesions included in the analysis were before, during or after genomic convergence. Despite the limited number of samples, the present study shows that reduced expression of mir15a in the blood is associated with OSCC staging.

\section{Acknowledgements}

This study was supported by grants from Fundação de Amparo à Pesquisa do Estado de Minas Gerais (FAPEMIG) and from Conselho Nacional de Desenvolvimento Científico e Tecnológico (CNPq), Brazil. Dr R.S. Gomez and Dr M.V. Gomez are research fellows of CNPq.

\section{References}

1. Chen CZ: MicroRNAs as oncogenes and tumor suppressors. N Engl J Med 353: 1768-1771, 2005.

2. Zhang B, Pan X, Cobb GP and Anderson TA: microRNAs as oncogenes and tumor suppressors. Dev Biol 302: 1-12, 2007.

3. Huppi K, Volfovsky N, Mackiewicz M, et al: MicroRNAs and genomic instability. Semin Cancer Biol 17: 65-73, 2007.

4. Kent OA and Mendell JT: A small piece in the cancer puzzle: microRNAs as tumor suppressors and oncogenes. Oncogene 25: 6188-6196, 2006.

5. Kumar MS, Lu J, Mercer KL, Golub TR and Jacks T: Impaired microRNA processing enhances cellular transformation and tumorigenesis. Nat Genet 39: 673-677, 2007.

6. Warnasulasuriya S: Global epidemiology of oral and oropharyngeal cancer. Oral Oncol 45: 309-316, 2009.

7. Gomes CC and Gomez RS: MicroRNA and oral cancer. Oral Oncol 44: 910-914, 2008.

8. Barnes L, Everson JW, Reichart P and Sidransky D: WHO World Health Organization Classification of Tumours, Pathology and Genetics of Head and Neck Tumours. IARC Press, Lyon, $\mathrm{p} 433,2005$.

9. Chen C, Ridzon DA, Broomer AJ, et al: Real-time quantification of microRNAs by stem-loop RT-PCR. Nucleic Acids Res 33: $1-9,2005$.

10. Livak KJ and Schimittgen T: Analysis of relative gene expression data using real-time quantitative PCR and the $2-\Delta \Delta \mathrm{Ct}$ method. Methods 25: 402-408, 2001
11. Tran N, McLean T, Zhang X, et al: MicroRNA expression profiles in head and neck cancer cell lines. Biochem Biophys Res Commun 358: 12-17, 2007.

12. Jiang J, Lee EJ, Gusev Y and Schimittgen TD: Real-time expression profiling of microRNA precursors in human cancer cell lines. Nucleic Acids Res 33: 5394-5403, 2005.

13. Gaur A, Jewell DA, Liang Y, et al: Characterization of microRNA expression levels and their biological correlates in human cancer cell lines. Cancer Res 67: 2456-2468, 2007.

14. Calin GA, Dumitru CD, Shimizu M, et al: Frequent deletions and down-regulation of micro-RNA genes miR 15 and miR16 at 13 q14 in chronic lymphocytic leukemia. Proc Natl Acad Sci USA 99: 15524-15529, 2002.

15. Cimmino A, Calin GA, Fabbri M, et al: miR-15 and miR-16 induce apoptosis by targeting BCL2. Proc Natl Acad Sci USA 102: 13944-13949, 2005.

16. Camisasca DR, Honorato J, Bernardo V, et al: Expression of Bcl-2 family proteins and associated clinicopathologic factors predict survival outcome in patients with oral squamous cell carcinoma. Oral Oncol 45: 225-233, 2009.

17. Ohigashi T, Ueno N, Novaka S, et al: Tyrosine kinase inhibitors reduce bcl-2 expression and induce apoptosis in androgendependent cells. Am J Physiol Cell Physiol 278: C66-C72, 2000.

18. Ogawara K, Miyakawa A, Shiba M, et al: Allelic loss of chromosome $13 \mathrm{q} 14.3$ in human oral cancer correlation with lymph node metastasis. Int J Cancer 79: 312-317, 1998.

19. Takamizawa J, Konishi H, Yanagizawa K, et al: Reduced expression of the let-7 microRNAs in human lung cancers in association with shortened postoperative survival. Cancer Res 64: 3753-3756, 2004.

20. Akao Y, Nakagawa Y and Naoe T: Let-7 microRNA functions as a potential growth suppressor in human colon cancer cells. Biol Pharm Bull 29: 903-906, 2006.

21. Childs G, Fazzari M, Kung G, et al: Low-level expression of microRNAs let-7d and mir-205 are prognostic markers of head and neck squamous cell carcinoma. Am J Pathol 174: 736-745, 2009.

22. Chang KW, Liu CJ, Chu TH, et al: Association between high mir-211 microRNA Expression and the poor prognosis of oral carcinoma. J Dent Res 87: 1063-1068, 2008.

23. Johnson SM, Grosshans H, Shingara J, et al: RAS is regulated by the let-7 microRNA family. Cell 120: 635-647, 2005.

24. Christensen BC, Moyer BJ, Avissar M, et al: Let-7 microRNA binging site polymorphism in the KRAS 3'UTR is associated with reduced survival in oral cancers. Carcinogenesis 30: 1003-1007, 2009.

25. Perez-Ordoñez B, Beauchemin M and Jordan RCK: Molecular biology of squamous cell carcinoma of the head and neck. J Clin Pathol 59: 445-453, 2006.

26. Hardisson D: Molecular pathogenesis of head and neck squamous cell carcinoma. Head Neck Oncol 260: 502-508, 2003.

27. Das BR and Nagpal JK: Understanding the biology of oral cancer. Med Sci Monit 8: 258-267, 2002.

28. Fukasawa K: Centrosome amplification, chromosome instability and cancer development. Cancer Lett 230: 6-19, 2005. 